\title{
MetaCRAST: Reference-guided extraction of CRISPR spacers from unassembled metagenomes
}

\author{
Abraham G Moller ${ }^{1}$, Chun Liang ${ }^{\text {Corresp. } 1}$ \\ ${ }^{1}$ Department of Biology, Miami University of Ohio, Oxford, Ohio, United States \\ Corresponding Author: Chun Liang \\ Email address: liangc@miamioh.edu
}

Clustered regularly interspaced short palindromic repeat (CRISPR) systems are the adaptive immune systems of bacteria and archaea against viral infection. While CRISPRs have been exploited as a tool for genetic engineering, their spacer sequences can also provide valuable insights into microbial ecology by linking environmental viruses to their microbial hosts. Despite this importance, metagenomic CRISPR detection remains a major challenge. Here we present a reference-guided CRISPR spacer detection tool (Metagenomic CRISPR Reference-Aided Search Tool - MetaCRAST) that constrains searches based on user-specified direct repeats (DRs). These DRs could be expected from assembly or taxonomic profiles of metagenomes. We compared the performance of MetaCRAST to those of two existing metagenomic CRISPR detection tools - Crass and MinCED - using both real and simulated acid mine drainage (AMD) and enhanced biological phosphorus removal (EBPR) metagenomes. Our evaluation shows MetaCRAST improves CRISPR spacer detection in real metagenomes compared to the de novo CRISPR detection methods Crass and MinCED. Evaluation on simulated metagenomes show it performs better than de novo tools for Illumina metagenomes and comparably for 454 metagenomes. It also has comparable performance dependence on read length and community composition, run time, and accuracy to these tools MetaCRAST is implemented in Perl, parallelizable through the Many Core Engine (MCE), and takes metagenomic sequence reads and direct repeat queries (FASTA or FASTQ) as input. It is freely available for download at https://github.com/molleraj/MetaCRAST. 
1 Classification: Microbiology, Ecology, Genomics, Bioinformatics, Computational Biology

2 Title: MetaCRAST: Reference-guided extraction of CRISPR spacers from

3 unassembled metagenomes

4 Abraham Moller ${ }^{1, \dagger}$ and Chun Liang ${ }^{1, *}$

5 1Department of Biology, Miami University, Oxford, Ohio 45056

$6 \quad$ *Corresponding Author: Chun Liang, liangc@miamioh.edu

7 tCurrent Address: Department of Microbiology and Immunology, Emory University,

8 Atlanta, Georgia 30322

9

10

11

12

13

14

15

16

17

18

19 


\section{Abstract}

Clustered regularly interspaced short palindromic repeat (CRISPR) systems are the adaptive immune systems of bacteria and archaea against viral infection. While CRISPRs have been exploited as a tool for genetic engineering, their spacer sequences can also provide valuable insights into microbial ecology by linking environmental viruses to their microbial hosts. Despite this importance, metagenomic CRISPR detection remains a major challenge. Here we present a reference-guided CRISPR spacer detection tool (Metagenomic CRISPR Reference-Aided Search Tool - MetaCRAST) that constrains searches based on user-specified direct repeats (DRs). These DRs could be expected from assembly or taxonomic profiles of metagenomes. We compared the performance of MetaCRAST to those of two existing metagenomic CRISPR detection tools - Crass and MinCED - using both real and simulated acid mine drainage (AMD) and enhanced biological phosphorus removal (EBPR) metagenomes. Our evaluation shows MetaCRAST improves CRISPR spacer detection in real metagenomes compared to the de novo CRISPR detection methods Crass and MinCED. Evaluation on simulated metagenomes show it performs better than de novo tools for Illumina metagenomes and comparably for 454 metagenomes. It also has comparable performance dependence on read length and community composition, run time, and accuracy to these tools MetaCRAST is implemented in Perl, parallelizable through the Many Core Engine (MCE), and takes metagenomic sequence reads and direct repeat queries (FASTA or FASTQ) as input. It is freely available for download at https://github.com/molleraj/MetaCRAST. 


\section{Introduction}

The clustered regularly interspaced short palindromic repeat (CRISPR) arrays found in prokaryotic genomes can help us better understand viral-microbial interactions important in many ecosystems. Viruses can release cellular nutrients back into the ecosystem through lytic infection, forming an ecological short-circuit called the viral shunt (Weitz \& Wilhelm, 2012). In this manner, viruses not only contribute to nutrient cycling in individual ecosystems, but also to maintaining biogeochemical cycles on a broader scale. The short spacers of viral DNA incorporated into CRISPR arrays form a historical record of past infections, thus linking virus to host (Sorek, Kunin \& Hugenholtz, 2008; Makarova, Wolf \& Koonin, 2013). This power of CRISPR spacers to determine viruses' host specificity has recently been exploited using metagenomes from many ecosystems (Anderson, Brazelton \& Baross, 2011; Sanguino et al., 2015; Edwards et al., 2015). While many tools exist for detecting CRISPRs in assembled genomes (Bland et al., 2007; Edgar, 2007; Grissa, Vergnaud \& Pourcel, 2007a; Rousseau et al., 2009), few exist for CRISPR detection in metagenomic reads (Rho et al., 2012; Skennerton, Imelfort \& Tyson, 2013; Skennerton).

The repetitive nature of CRISPRs makes them difficult to assemble from metagenomes, necessitating special tools to detect them in unassembled reads. Several tools have been developed to detect and assemble CRISPR arrays in unassembled reads

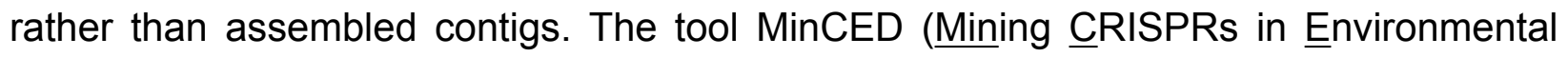
Datasets), like metaCRT (Rho et al., 2012), is a modified version of CRT (Bland et al., 2007) that detects CRISPR spacers (Skennerton), while the tool Crass (CRISPR assembler) detects and assembles CRISPR arrays (Skennerton, Imelfort \& Tyson, 2013), 
both from raw metagenomic reads. MinCED searches each read for CRISPRs using the same strategy as CRT; it searches for appropriately spaced short k-mers from which it extends longer repeats if appropriately frequent nucleotides are identified at the ends of the growing repeats. Crass relies on a hybrid algorithm to detect spacers that blends strategies of CRT (Bland et al., 2007) and CRISPRFinder (Grissa, Vergnaud \& Pourcel, 2007b). In long reads (>177 bp), it searches for repeats using the CRT strategy previously described. In short reads ( $<177 \mathrm{bp})$, on the other hand, it searches for appropriately spaced full-length repeats (i.e., 20-50 bp) and extends these repeats only with identical nucleotides, thus avoiding the potential errors caused by the CRT algorithm over- or under-extending the few repeats found in a short sequence. Crass then searches further for reads containing a single repeat, determines consensus direct repeats, uses the first and last k-mers of detected spacers to build a graph of spacer arrangement, and assembles CRISPR arrays using this graph. Both MinCED and Crass do not rely on prior knowledge of direct repeat sequences, making them de novo detection methods. Instead, they use heuristics to determine whether detected repeats are indeed CRISPRs. Such heuristics include threshold array lengths to avoid short, spurious CRISPR arrays and threshold repeat-spacer similarities to avoid arrays where spacers are too similar to repeats (Bland et al., 2007; Grissa, Vergnaud \& Pourcel, 2007a; Skennerton, Imelfort \& Tyson, 2013), which might indicate microsatellites rather than CRISPRs.

In this work, we present the Metagenomic $\underline{\text { CRISPR }}$ Reference-Aided Search $\underline{\text { Tool }}$ (MetaCRAST), a novel reference-guided tool to improve CRISPR spacer detection in unassembled metagenomic sequencing reads. While MetaCRAST, to our knowledge, is the first reference-guided, read-dependent metagenomic CRISPR detection tool, prior 
studies have used known direct repeats to improve CRISPR detection. The genomic CRISPR identification algorithm CRISPRDetect matches newly identified direct repeats to a reference library to refine repeat boundaries and validate arrays (Biswas et al., 2016). Searching reference repeat libraries, together with annotating cas genes adjacent to CRISPR arrays, has been used to exclude false positive "putative" CRISPRs from CRISPR annotation (Zhang \& Ye, 2017). Unlike MinCED and Crass, as a referenceguided method, MetaCRAST constrains spacer detection by searching metagenomes for direct repeats (DRs) that the user specifies. Relationships amongst these tools and such differences in use are further illustrated in Figure 1. Such specified DRs may be selected based on assembly or taxonomic profiling of metagenomic reads. MetaCRAST improves CRISPR annotation by allowing users to control for the taxonomic composition of the metagenome. It also avoids the rejection of true CRISPRs that can occur due to the heuristics required for de novo detection methods. In addition, unlike Crass and MinCED, MetaCRAST provides consistent performance over different read length Illumina datasets.

\section{Materials and Methods}

\section{Algorithm and implementation}

MetaCRAST can constrain spacer detection by expected host species' DRs or DRs identified from assembly (Figure 2A). It searches each read for DR sequences matching query DRs specified by the user. These DRs can be selected from CRISPR arrays detected with genomic CRISPR detection tools such as PILER-CR (Edgar, 2007), CRF (Wang \& Liang, 2017), or CRISPRFinder (Grissa, Vergnaud \& Pourcel, 2007b) in fully assembled microbial genomes or assembled metagenomic contigs. The steps of the 
112 MetaCRAST pipeline are outlined in Figure 2B. In the first step of the pipeline, reads

113 containing DRs within a certain Levenshtein edit distance (i.e., number of insertions,

114 deletions, or substitutions necessary to convert one sequence to another) of the query

115 DRs are quickly identified using the Wu-Manber multi-pattern search algorithm (Wu,

116 Manber \& Myers, 1995). In the second step, individual reads found to contain a query DR

117 sequence are searched for two or more copies of the query DRs. In the third step, the

118 sequence fragments between the DRs detected in these sequence reads are extracted

119 into a comprehensive spacer set, which are then clustered using CD-HIT into a non-

120 redundant unique spacer set stored in FASTA format (Li \& Godzik, 2006, p.).

MetaCRAST is implemented in Perl as a command line tool to analyze

122 metagenomes in FASTA or FASTQ formats. The tool has been implemented in several

123

124

125

126

127

128

129

130

131

132

133

versions that differ in metagenome loading method (using BioPerl or readfq, the latter of which was paired either with the standard open routine to load a single file or mce_open for parallel file loading). Optionally, the user can specify the maximum spacer length, the distance metric used for comparing DRs to reads (Hamming or Levenshtein), whether to search for the reverse complement of the DR, the CD-HIT similarity threshold for clustering spacers, and the maximum number of threads to use to parallelize the search. The reverse complement argument (-r) should be used when the CRISPR direction is unknown. When the search is run in parallel, the FASTA (or FASTQ) file is split based on the specified number of threads. All command line arguments are further described in Table 1. Each split file is searched in parallel. An additional tool has been provided to assist taxonomy-guided query selection. This tool searches a taxonomically-annotated 
134 library of CRISPRdb DRs for those that belong to a particular taxon query (e.g., 135 Streptococcus).

136 To analyze the distribution of taxonomic affiliations to direct repeats, we examined

137 all direct repeats found in microbial genomes using the CRISPRdb database. CRISPRdb 138 provides a library of direct repeats labeled with respective GenBank accessions in the 139 CRISPR utilities section of the database (Grissa, Vergnaud \& Pourcel, 2007a). We 140 processed this library to assign taxonomy information based on GenBank accession. 141 Taxonomy information was extracted from GenBank records with the Perl module 142 Bio::DB::GenBank. Statistics describing the distribution of unique binomial names or 143 genuses to which individual direct repeats affiliated was compiled with Microsoft Excel. 144 Binomial name (species-level) and genus statistics are presented in Table 2.

\section{Performance evaluation with simulated and real metagenomes}

To study the relationship between CRISPR spacer detection and read length or sequencing technology, simulated acid mine drainage (AMD) and enhanced biological phosphorus removal (EBPR) metagenomes were generated using Grinder (Angly et al., 2012). We generated simulated metagenomes over a range of average read lengths (100 to 600 base pairs) using models of 454 (Balzer et al., 2010) and Illumina (Korbel et al., 2009) errors. Following previous studies, we used a fourth-degree polynomial (3e-3 + $3.3 e-8 *{ }^{\wedge} 4$, where $i$ is the nucleotide position from the 5 ' end of the read, and the output is percentage chance of an error at that position) to model the Illumina sequencing error 154 rate (Dohm et al., 2008; Korbel et al., 2009; Angly et al., 2012). This polynomial determined the probability of substitution, insertion, or deletion at each base of a 156 simulated read (Korbel et al., 2009). For Illumina simulations, the ratio of substitutions to 
157 insertions and deletions was set to $80: 20$ by default. For 454 metagenome simulations,

158 we modeled homopolymer errors as homopolymer length variation within simulated

159 reads. The distributions of homopolymer lengths were defined by the mean $\mathrm{n}$ and 160 standard deviation $0.03494+n * 0.06856$, where $n$ is the homopolymer length, based on 161 a prior study (Balzer et al., 2010; Angly et al., 2012).

We generated six simulated metagenomes per condition (average read length, model, and microbial community). We used highly simplified taxonomic profiles to model the AMD and EBPR metagenomes (Tables S1 and S2). To test the effects of community composition on spacer detection, we simulated the AMD metagenome with a 454 error model and $600 \mathrm{bp}$ average read length, varying the relative proportions of Leptospirillum and Ferroplasma genome used for the simulation (i.e., from 0 to 100\% Leptospirillum). All simulated metagenomes contained 100,000 reads. 454 metagenomes were 169 generated with this command: grinder -reference_file AMDgenomes.fasta abundance_file AMDprofile.txt -total_reads 100000 -read_dist (one of 100, 150, 200, 250, 300, 400, or 600) normal 50 -homopolymer_dist balzer. All 454 read length distributions were normal with a standard deviation of $50 \mathrm{bp}$. Illumina metagenomes were generated 173 with this command: grinder -reference_file AMDgenomes.fasta -abundance_file 174 AMDprofile.txt -total_reads 100000 -read_dist (one of 100,150, 200, 250, or 300) -md poly4 3e-3 3.3e-8. All Illumina read length distributions were uniform with all reads having exactly the average read length.

Simulated metagenomes were searched for CRISPR spacers using Crass 178 (Skennerton, Imelfort \& Tyson, 2013), MinCED (Skennerton), and MetaCRAST. Crass and MinCED were run with default parameters (crass grinder-reads.fa; minced -spacers 
180 grinder-reads.fa minced.crispr). The default minimum and maximum DR lengths for both

181 Crass and MinCED were 23 and $47 \mathrm{bp}$. The default minimum and maximum spacer

182 lengths for both Crass and MinCED were 26 and $50 \mathrm{bp}$. MetaCRAST was run with a 183 taxonomy-guided query (Tables S3 and S4), a maximum spacer length of 60 , a maximum 184 allowed edit distance (insertions, deletions, or substitutions) between query and target 185 direct repeats of 3 , a CD-HIT clustering similarity threshold of 0.9 , and a total of 16 parallel 186 threads (MetaCRAST -p query.fa -i grinder-reads.fa -o MetaCRAST -d 3 -I 60 -c 0.90 -a $1870.90-n 16$-t tmp). We selected a maximum allowed edit distance of 3 based on results 188 of our prior metagenomic CRISPR detection studies, which showed MetaCRAST 189 searches with a taxonomy-guided query found similar numbers of spacers to Crass when we set this edit distance (Moller \& Liang, 2017). For all analyses, detected spacers were clustered with CD-HIT with a similarity threshold of 0.9 (cdhit $-i$ spacers.fa -0 spacers $C D 90 . f a-c$ 0.9) to reduce spacer redundancy. Performance on these simulated metagenomes was evaluated based on total number of spacers detected, number of false positive spacers detected, and run time for each average read length. For the mixed composition simulated AMD metagenomes described above, spacers were aligned against CRISPR spacers present in the source Leptospirillum and Ferroplasma genomes and the number of matching true positive spacers for each organism reported.

The number of false positive spacers found in simulated metagenomes was determined by comparing the total detected spacers with the expected CRISPRdb spacers found in the source genomes used for the simulations (AMD and EBPR). Alignments were made to the annotated CRISPRdb spacers using BLAST with an E- 
203 cutoff of $1 \mathrm{e}-1$ to consider whether the original threshold was too stringent. The number

204 ofdetected spacers that were aligned to expected ones was subtracted from the total

205 number of spacers detected to determine the number of false positive spacers for a 206 particular method and condition. Cases where zero spacers were detected in a 207 metagenome were treated as zero false positive spacers and included in overall analysis.

208 Run times were determined for each metagenome and method using the built-in Linux 209 command time. Run time was calculated as the sum of the user and system time (together 210 the total CPU time).

Similarly, CRISPR spacers were also detected by the aforementioned three tools in real AMD and EBPR metagenomes (Table S5) downloaded from iMicrobe (Hurwitz, 213 2014) and taxonomically profiled with MetaPhyler (Liu et al., 2011). MetaCRAST analyses of the real metagenomes were performed with taxonomy- or assembly-guided query DRs generated as follows. To make an assembly-guided query, CAP3-assembled contigs (Huang \& Madan, 1999) were searched for CRISPR DRs using PILER-CR (Edgar, 2007), which finds CRISPRs in assembled genomes or contigs. These DRs formed an assembly-guided query (Tables S6 and S7), while DRs found in assembled Leptospirillum (AMD), Ferroplasma (AMD), and Candidatus Accumulibacter phosphatis (EBPR) genomes included in CRISPRdb (Grissa, Vergnaud \& Pourcel, 2007a) formed a to be major components of the microbial community based on the AMD and EBPR taxonomic profiles determined with MetaPhyler (Tables S8 and S9). 


\section{Results}

227 Effects of read length, sequencing technology, and community composition on 228 CRISPR spacer detection

We first investigated the relationships between detected spacers and read length or sequencing technology. Performance, here determined by the number of spacers detected, increased with read length over all 454 tests (Figure 3). While the total number of spacers detected by Crass and MetaCRAST converged as read length increased, the total number of spacers detected by MinCED steadily increased even beyond the true number of spacers found in the genomes used to generate the simulated metagenomes. We speculate that MinCED inconsistently determined DR lengths amongst different CRISPR-containing reads due to its CRT-based algorithm, leading to the same spacers being inappropriately truncated or extended. Meanwhile, amongst metagenomes simulated with the Illumina model, MetaCRAST detected significantly more spacers than Crass and MinCED for average read lengths of 200 bp or greater (Figure 3; $p<0.05$ for both AMD and EBPR simulations using unpaired t-tests). Crass detected more spacers than MinCED and MetaCRAST for short Illumina reads (100 and $150 \mathrm{bp}$ ), however (Figure 3; $\mathrm{p}<0.05$ for both AMD and EBPR simulations using unpaired t-tests).

We also tested the effects of community composition on CRISPR detection for each of the three methods using AMD metagenomes simulated with a 454 error model and $600 \mathrm{bp}$ average read length. We selected the $600 \mathrm{bp}$ average read length for all mixed metagenomes to minimize differences in detection between methods based on

read length (Figure 3). We varied the relative abundances of Leptospirillum and

248 Ferroplasma from 0 to 100 percent in our taxonomic profiles, thus varying the proportions 
249 of CRISPR arrays specific to each included in the simulated metagenomes. For all

250 detection methods, detected spacers specific to a genome decreased as the relative

251 proportion of that taxon decreased, with roughly the same pattern for each method (Figure

252 4). As in the read length studies, MinCED consistently detected far more genome-specific

253 spacers in the metagenomes than were originally present in the source genomes (Figure

254 4). This may account for its steeper increase in detected genome-specific spacers as the

255 proportion of the corresponding genome in the simulated metagenomes increased.

256 Evaluation of CRISPR spacer detection on real AMD and EBPR metagenomes

We also evaluated MetaCRAST against Crass and MinCED using real AMD and

258

259

260

261

262

263

264

265

266

267

268

269

270

271 queries consistently found fewer spacers than the other two methods (583 compared to 2486 for Crass and 4265 for MinCED in the AMD metagenome; 196 compared to 1014 for Crass and 1821 for MinCED in the EBPR metagenome), an assembly-guided MetaCRAST search identified more spacers than Crass did in the AMD metagenome (2813 compared to 2486 - Figure 5A). In both AMD and EBPR metagenomes, many common spacers were detected amongst Crass, MetaCRAST (assembly-guided query), and MinCED (7.1\% of all detected spacers for AMD and 2.5\% for EBPR - Figures 5B and 5C). Despite this, there were also many spacers detected with Crass and MinCED not identified with MetaCRAST searches (Figures 5B and 5C). Notably, however, none of the spacers detected with MetaCRAST using the taxonomy-guided query overlapped with the Crass-detected spacers (Figures 5B and 5C), suggesting MetaCRAST can detect spacers missed by Crass given an appropriate taxonomy-guided query. 
272 Evaluation of accuracy and runtime performance

273

274

275

276

277

278

279

280

281

282

283

284

285

286

287

288

289

290

291

292

293

294

In addition to our studies comparing detected spacers over a variety of conditions, we evaluated all three detection methods for spacer detection accuracy and run time (Figures 6 and 7). We performed these evaluations on the simulated AMD and EBPR metagenomes previously used to examine effects of read length and sequencing technology on CRISPR detection (Figure 3). For AMD metagenomes simulated with the 454 model, MinCED detected significantly more false positive spacers than Crass or MetaCRAST for average read lengths of 200 bp or more (Figure 6; $p<0.05$ using unpaired t-tests). Crass and MetaCRAST, on the other hand, did not have statistically significant differences in detected false positive spacers over the entire range of average read lengths ( $p>0.05$ using unpaired t-tests). For the AMD Illumina metagenomes, on the other hand, MetaCRAST generated the largest number of false positive spacers for average read lengths greater than $200 \mathrm{bp}$ (Crass for average read lengths of $150 \mathrm{bp}$ and lower), but not by a statistically significant margin compared with MinCED ( $p>0.05$ using unpaired t-tests). For the EBPR metagenomes simulated with the 454 model, there were remarkably few false positive spacers detected with all methods over the full range of average read lengths. For the EBPR Illumina metagenomes, MinCED generated the largest number of false positive spacers for average read lengths greater than 200 bp (Crass for average read lengths of $150 \mathrm{bp}$ and lower), with MetaCRAST overlapping its pattern closely (Figure 6). Because of this overlap, differences between MinCED and MetaCRAST false positive spacers were not statistically significant $(p>0.05$ using unpaired t-tests), (EBPR Illumina metagenomes, Figure 6). MetaCRAST did detect more false positives than MinCED for the 200 bp read length $(p<0.05$ using unpaired t-tests, 
295 EBPR Illumina metagenomes, Figure 6). We note that these false positive spacers are

296 only detected spacers that did not align to expected ones. The false positives do not

297

298

299

300

301

302

303

304

305

306

307

308

309

310

311

312

313

314

315

316

317

necessarily include improperly truncated or extended spacers, which we suspect MinCED creates, leading to its artificially high spacer counts (Figure 3 ). We repeated this false positive spacer analysis using a weaker E-value threshold of 1e-1 (Figure S1). Using this weaker threshold decreased the number of false positive spacers identified in all conditions (Figure S1).

We also evaluated relative speed of the detection methods using the Linux function time. We evaluated seven different combinations of algorithms, implementations, and parameters. We evaluated both Crass and MinCED with default parameters. For MetaCRAST, we evaluated five different conditions differing in parallelization and metagenome loading method - BioPerl for loading and 16 threads, BioPerl and a single thread, readfq with mce_open for loading and 16 threads, readfq with mce_open and a single thread, and readfq with the standard open routine and a single thread (Figure 7). We used CPU time (user and system time) rather than wall clock time (real time) as a measure of speed performance.

We noticed steady increases in run time with increasing read length for all detection methods, metagenomes, and sequencing technologies (Figure 7). MetaCRAST showed a linear CPU time dependence on read length in all cases $\left(R^{2}>0.98\right.$ in all cases; p-values calculated from Pearson correlation were less than 1e-5 in all cases), while linear correlations for MinCED and crass were much weaker $\left(R^{2}<0.88\right.$ in all cases; $p$ values calculated from the Pearson correlations were more than 0.05 for Illumina datasets but between $9 \mathrm{e}-4$ and $8 \mathrm{e}-3$ for 454 datasets). Among MetaCRAST implementations, the 
318 readfq/open version used the least CPU time by statistically significant margins for all

319 conditions (Figure 7; $p<0.05$ in all cases using unpaired t-tests). MetaCRAST was slower

320 than Crass for all read lengths by statistically significant margins (Figure 7; $p<0.05$ in all

321 cases using unpaired t-tests). On the other hand, it was faster than MinCED for 454 read

322 lengths between 100 and 400 bp and Illumina read lengths between 100 and 250 bp

323 (Figure 7; $p<0.05$ using unpaired t-tests).

324

325

326

327

328

329

330

331

332

333

334

335

336

337

338

339

\section{Taxonomic affiliations of CRISPR direct repeats annotated in CRISPRdb}

To analyze how direct repeats affiliated to taxa, we examined all direct repeats annotated in microbial genomes using the CRISPRdb database. We used a Perl script to assign taxonomy information based on GenBank accession using the module Bio::DB::GenBank. The results of this analysis for species (binomial name) and genuslevel designations are presented in Table 2. The average number of unique taxon designations per DR was greater at the species level than the genus level (1.308 compared to 1.063). Variation was also greater for species-level designations compared to genus-level (standard deviation of 1.567 compared 0.521 ). Both species- and genuslevel analyses identified DRs that were affiliated to many taxa (a maximum of 20 genuses and 46 species). We acknowledge that our analysis does not examine the number of unique DRs per taxon. It also only considers independent, unique DRs, ignoring the possibility that many unique DRs may have closely related sequences.

\section{Discussion}

In this work, we present and evaluate a novel reference-guided method for CRISPR detection in unassembled metagenomic reads. This method searches 
340 metagenomic reads for user-specified direct repeats which could be provided through

341 taxonomy- or assembly-guided searches (Figures 1 and 2). We analyzed currently known

342 DRs with respect to their taxonomic designations to determine the robustness of

343 taxonomy-guided searches (Table 2). We found that most DRs in fact do affiliate to a

344 single species or genus, but that there are exceptions that may have arisen through

345 horizontal gene transfer (Table 2). This analysis does not consider small polymorphisms

346 between closely related DRs. Depending on the circumstance, it may be important to

347 consider whether one DR could be present in multiple taxa found in a sample.

Our studies of simulated metagenomes show distinct advantages for Crass and

MetaCRAST depending on average read length (Figure 3). While the modified assembly procedure and exhaustive searches Crass provides make it well suited for short read 454 and Illumina metagenomes, MetaCRAST outperforms Crass for long read Illumina metagenomes (Figure 3). We speculate that heuristics to avoid misassembly of CRISPR arrays or improper repeat detection may hinder Crass in these long-read Illumina metagenomes. We also noted that all three algorithms detected far more spacers in 454 compared to Illumina metagenomes (Figure 3). We have two possible explanations for this phenomenon. First, our algorithms may have handled homopolymer error better than

357 the substitution error simulated in the Illumina metagenomes. Second, our Illumina model may have introduced higher error rates than the 454 error model, making it more difficult

359 to find multiple similar DRs in the reads. The very high numbers of MinCED-detected spacers are deceptive because this algorithm has the potential for substantial errors in

361 determining repeat and spacer lengths (Figures 3 and 4). Inconsistencies in defining 362 repeat length leads to false splitting of identical spacers into different groups. 
Studies on real metagenomes suggest substantial advantages for Crass and MinCED in terms of numbers of detected spacers (Figure 5). While in most cases MetaCRAST detected fewer spacers than Crass or MinCED, it did identify spacers unique to those from the two other methods. This suggests that it can complement these methods, finding spacers missed due to the heuristics that Crass and MinCED use to avoid false positives (Figure 5). We had expected that MetaCRAST would underperform compared to Crass and MinCED in these real metagenomes, because the taxonomyguided queries we used did not fully account for all the taxa found with taxonomic profiling.

371 We only used one or two genomes to simulate the AMD and EBPR metagenomes,

372 making the simulated metagenomes much simpler in taxonomic diversity. This

373 simplification was what made MetaCRAST detection performance comparable to that of 374 Crass and MinCED for the simulations.

Accuracy was roughly similar amongst the three tools (Figure 6). Relaxing the error threshold reduced false positive spacers detected by all tools, suggesting sequencing error rather than algorithm issues could account for some of these false positive spacers (Figure S1). MetaCRAST follows the same pattern of increasing run time with average read length as the other two tools, and it is comparable in run time to MinCED (Figure 7). MetaCRAST run time increases linearly with average read length (Figure 7). We acknowledge that implementation of the algorithm in a compiled language or increasing the number of threads used to parallelize the search could further improve MetaCRAST speed. Nonetheless, while MetaCRAST is not as fast as the compiled algorithm Crass under the conditions tested, it does identify spacers distinct from these methods in real 
385 metagenomes and outperforms it in overall spacer detection for simulated Illumina 386 metagenomes.

Recent studies of computational methods for determining phage-host interactions suggest CRISPR spacer alignment is a highly accurate signature of phage-host interaction but that most identified CRISPR spacers do not align to known phage genomes (Edwards et al., 2015). This suggests that it is critical to improve metagenomic CRISPR spacer detection to increase the chances of matching spacers to viral genomes. More broadly, increasing spacer matching would provide a fuller appreciation of a microbial ecosystem's phage-host interaction space. We have recently used MetaCRAST to improve our determination of virus-host interactions in solar salterns (Moller \& Liang, 395 2017), complementing Crass with our spacer detection method. MetaCRAST complements de novo methods like Crass because it avoids the heuristics they use to reduce false positive spacers. Using a targeted direct repeat query, our tool can avoid the false negative bias of these approaches. We anticipate that MetaCRAST will be of great interest to microbial ecologists interested in phage-host interactions because it complements existing de novo methods to improve metagenomic CRISPR detection.

\section{Acknowledgements}

Thanks to Michael Crowder and Gary Lorigan (Miami University) for feedback on the project and manuscript. 


\section{References}

408 Altschul SF., Gish W., Miller W., Myers EW., Lipman DJ. 1990. Basic local alignment

409

410

411

412

413

414

415

416

417

418

419

420

421

422

423

424

425

426

427 search tool. Journal of Molecular Biology 215:403-410. DOI: 10.1016/S00222836(05)80360-2.

Anderson RE., Brazelton WJ., Baross JA. 2011. Using CRISPRs as a metagenomic tool to identify microbial hosts of a diffuse flow hydrothermal vent viral assemblage: CRISPR spacers reveal hosts of marine vent viral assemblage. FEMS Microbiology Ecology 77:120-133. DOI: 10.1111/j.1574-6941.2011.01090.x.

Angly FE., Willner D., Rohwer F., Hugenholtz P., Tyson GW. 2012. Grinder: a versatile amplicon and shotgun sequence simulator. Nucleic Acids Research 40:e94-e94. DOI: $10.1093 /$ nar/gks251.

Balzer S., Malde K., Lanzén A., Sharma A., Jonassen I. 2010. Characteristics of 454 pyrosequencing data-enabling realistic simulation with flowsim. Bioinformatics 26:i420-i425. DOI: 10.1093/bioinformatics/btq365.

Biswas A., Staals RHJ., Morales SE., Fineran PC., Brown CM. 2016. CRISPRDetect: A flexible algorithm to define CRISPR arrays. BMC Genomics 17:356. DOI: 10.1186/s12864-016-2627-0.

Bland C., Ramsey TL., Sabree F., Lowe M., Brown K., Kyrpides NC., Hugenholtz P. 2007. CRISPR Recognition Tool (CRT): a tool for automatic detection of clustered regularly interspaced palindromic repeats. BMC Bioinformatics 8:209. DOI: $10.1186 / 1471-2105-8-209$. 
428 Dohm JC., Lottaz C., Borodina T., Himmelbauer H. 2008. Substantial biases in ultra429 short read data sets from high-throughput DNA sequencing. Nucleic Acids Research 36:e105. DOI: 10.1093/nar/gkn425.

431 Edgar RC. 2007. PILER-CR: Fast and accurate identification of CRISPR repeats. BMC Bioinformatics 8:18. DOI: 10.1186/1471-2105-8-18.

433

434

435

436

437

438

439

440

441

442

443

444

445

446

447

448

449 approaches to predict bacteriophage-host relationships. FEMS Microbiology Reviews:fuv048. DOI: 10.1093/femsre/fuv048.

Grissa I., Vergnaud G., Pourcel C. 2007a. The CRISPRdb database and tools to display CRISPRs and to generate dictionaries of spacers and repeats. BMC Bioinformatics 8:172. DOI: 10.1186/1471-2105-8-172.

Grissa I., Vergnaud G., Pourcel C. 2007b. CRISPRFinder: a web tool to identify clustered regularly interspaced short palindromic repeats. Nucleic Acids Research 35:W52-W57. DOI: 10.1093/nar/gkm360.

Huang X., Madan A. 1999. CAP3: A DNA Sequence Assembly Program. Genome Research 9:868-877. DOI: 10.1101/gr.9.9.868.

Hurwitz B. 2014. iMicrobe: Advancing Clinical and Environmental Microbial Research using the iPlant Cyberinfrastructure. In: Plant and Animal Genome,.

Korbel JO., Abyzov A., Mu XJ., Carriero N., Cayting P., Zhang Z., Snyder M., Gerstein MB. 2009. PEMer: a computational framework with simulation-based error models for inferring genomic structural variants from massive paired-end sequencing data. Genome Biology 10:R23. DOI: 10.1186/gb-2009-10-2-r23. 
450 Li W., Godzik A. 2006. Cd-hit: a fast program for clustering and comparing large sets of 451 protein or nucleotide sequences. Bioinformatics 22:1658-1659. DOI:

452

453

454

455

456

457

458

459

460

461

462

463

464

465

466

467

468

469

470

Martín HG., Ivanova N., Kunin V., Warnecke F., Barry KW., McHardy AC., Yeates C., He S., Salamov AA., Szeto E., Dalin E., Putnam NH., Shapiro HJ., Pangilinan JL., Rigoutsos I., Kyrpides NC., Blackall LL., McMahon KD., Hugenholtz P. 2006. Metagenomic analysis of two enhanced biological phosphorus removal (EBPR) sludge communities. Nature Biotechnology 24:1263-1269. DOI: $10.1038 / \mathrm{nbt} 1247$.

Moller AG., Liang C. 2017. Determining virus-host interactions and glycerol metabolism profiles in geographically diverse solar salterns with metagenomics. PeerJ 5:e2844. DOI: 10.7717/peerj.2844.

Rho M., Wu Y-W., Tang H., Doak TG., Ye Y. 2012. Diverse CRISPRs Evolving in Human Microbiomes. PLoS Genetics 8:e1002441. DOI: 10.1371/journal.pgen.1002441. 
471 Rousseau C., Gonnet M., Romancer ML., Nicolas J. 2009. CRISPI: a CRISPR

472

473

474

475

476

477

478

479

480

481

482

483

484

485

486

487

488

489

490

491

492 interactive database. Bioinformatics 25:3317-3318. DOI:

10.1093/bioinformatics/btp586.

Sanguino L., Franqueville L., Vogel TM., Larose C. 2015. Linking environmental prokaryotic viruses and their host through CRISPRs. FEMS Microbiology Ecology 91:fiv046. DOI: 10.1093/femsec/fiv046.

Skennerton C.minced - Mining CRISPRs in Environmental Datasets. Available at https://github.com/ctSkennerton/minced (accessed May 27, 2016).

Skennerton CT., Imelfort M., Tyson GW. 2013. Crass: identification and reconstruction of CRISPR from unassembled metagenomic data. Nucleic Acids Research 41:e105-e105. DOI: 10.1093/nar/gkt183.

Sorek R., Kunin V., Hugenholtz P. 2008. CRISPR - a widespread system that provides acquired resistance against phages in bacteria and archaea. Nature Reviews Microbiology 6:181-186. DOI: 10.1038/nrmicro1793.

Tyson GW., Chapman J., Hugenholtz P., Allen EE., Ram RJ., Richardson PM., Solovyev VV., Rubin EM., Rokhsar DS., Banfield JF. 2004. Community structure and metabolism through reconstruction of microbial genomes from the environment. Nature 428:37-43. DOI: 10.1038/nature02340.

Wang K., Liang C. 2017. CRF: detection of CRISPR arrays using random forest. PeerJ 5:e3219. DOI: 10.7717/peerj.3219.

Weitz JS., Wilhelm SW. 2012. Ocean viruses and their effects on microbial communities and biogeochemical cycles. F1000 Biology Reports 4. DOI: 10.3410/B4-17. 
493 Wu S., Manber U., Myers E. 1995. A Subquadratic Algorithm for Approximate Regular 494 Expression Matching. Journal of Algorithms 19:346-360. DOI:

495 10.1006/jagm.1995.1041.

496 Zhang Q., Ye Y. 2017. Not all predicted CRISPR-Cas systems are equal: isolated cas

497 genes and classes of CRISPR like elements. BMC Bioinformatics 18:92. DOI:

498 $10.1186 / \mathrm{s} 12859-017-1512-4$.

499

500

501

502

503

504

505

506

507

508

509

510

511

512 


\section{Figures}

514 Figure 1: This diagram outlines relationships amongst different metagenomic CRISPR

515 detection methods. CRISPR detection can be performed either using specified direct

516 repeats (reference-guided detection) or without prior knowledge of direct repeat

517 sequences (de novo detection). De novo detection searches raw metagenomic reads for

518 direct repeat sequences of the appropriate length and spacing (i.e., 25-60 bp long repeats

519 with 25-60 bp spacers between them). De novo detection techniques either detect 520 spacers in reads only (MinCED) or assemble reads into arrays (Crass). Reference-guided

521 CRISPR detection, on the other hand, searches reads for user-specified direct repeat

522 sequences, and extracts spacers from between direct repeat sequences identified in

523 reads containing direct repeats. While the query is user-specified, general strategies for

524 generating a query include using direct repeats found in assembled metagenomic contigs

525 with CRISPR array detection tools (e.g., PILER-CR) or direct repeats found in genomic

526 CRISPR arrays (e.g., those found in microbial genomes included in CRISPRdb) that

527 might be expected based on taxonomic profiles. An example of the latter strategy would

528 be searching for known genomic Streptococcus pyogenes direct repeats if Streptococcus

529 pyogenes is found in the metagenome's taxonomic profile.

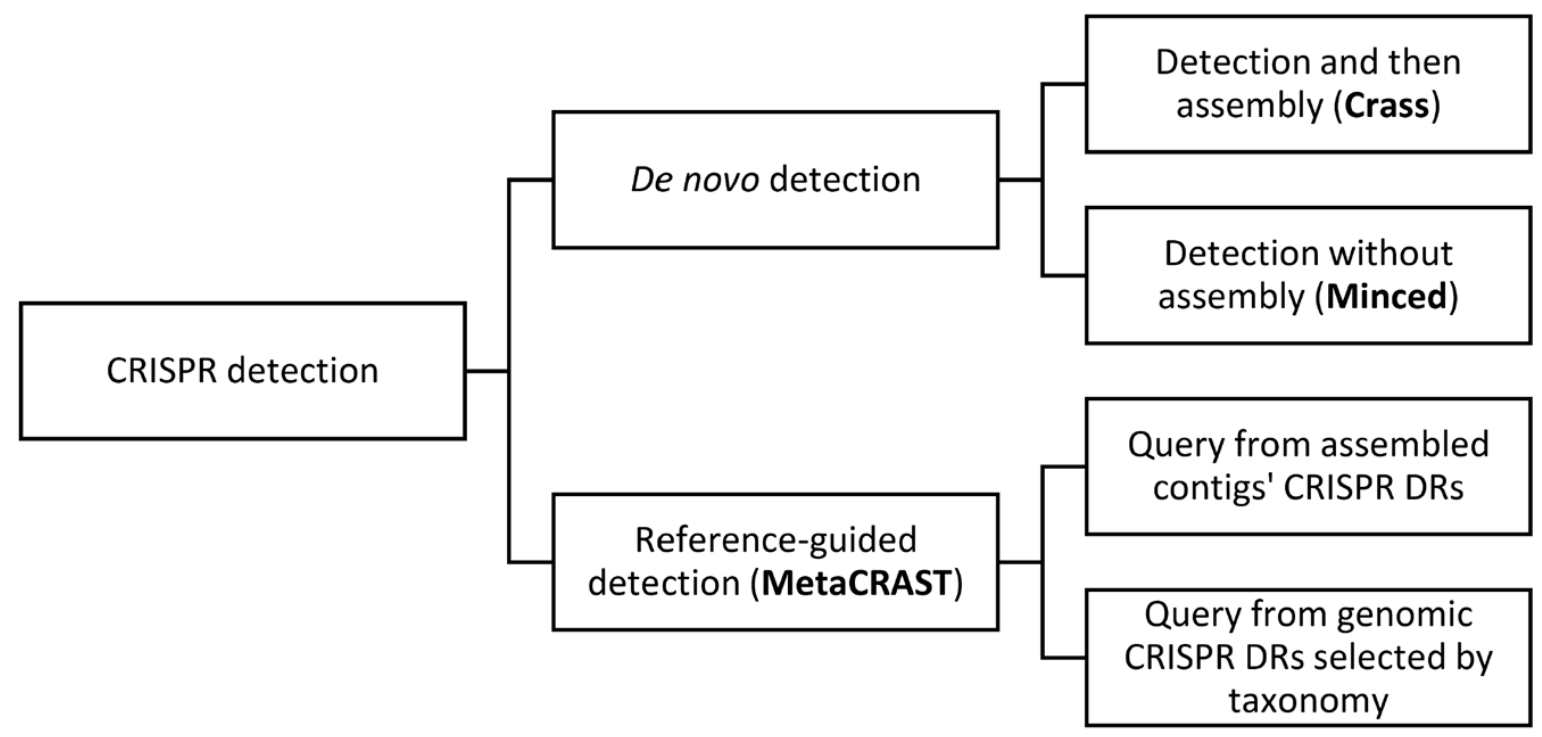


532 Figure 2: A comparison of per-read CRISPR detection strategies (A) between

533 MetaCRAST and existing de novo detection tools (e.g., Crass, MinCED) and an outline 534 of the MetaCRAST workflow (B). DR represents direct repeat, while $S$ represents spacer.

A

Known DR

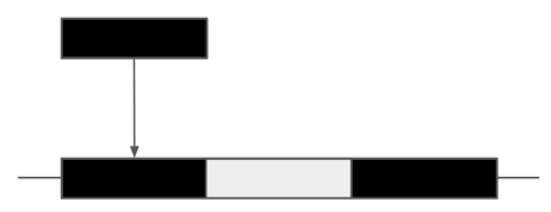

Known DR S Known DR

Reference-guided detection
Look for any new DRs:

Find new DR pair in read

Appropriately spaced

"Seed"

read

DR $\quad S \quad$ DR

De novo detection

(Crass, minced)

B

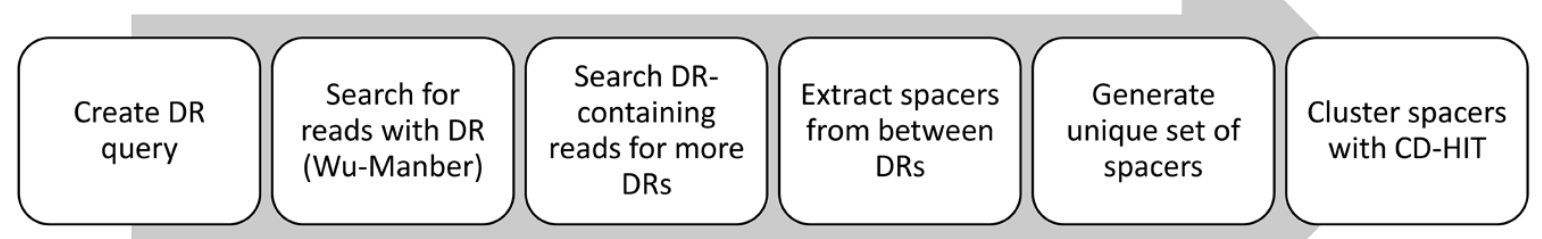


542 Figure 3: Evaluation of MetaCRAST, Crass, and MinCED performance on simulated AMD 543 ( $A$ and $B$ ) and EBPR ( $C$ and $D$ ) metagenomes. The procedure used to generate the 544 simulated metagenomes is described in Materials and Methods. All data points represent 545 the averages of six individual simulations and are presented with error bars representing 546 two times the standard error above and two below the average. The true number of 547 spacers expected in each simulated metagenome is marked with a black line (138 548 expected in the AMD metagenomes; 219 in the EBPR metagenomes).

549

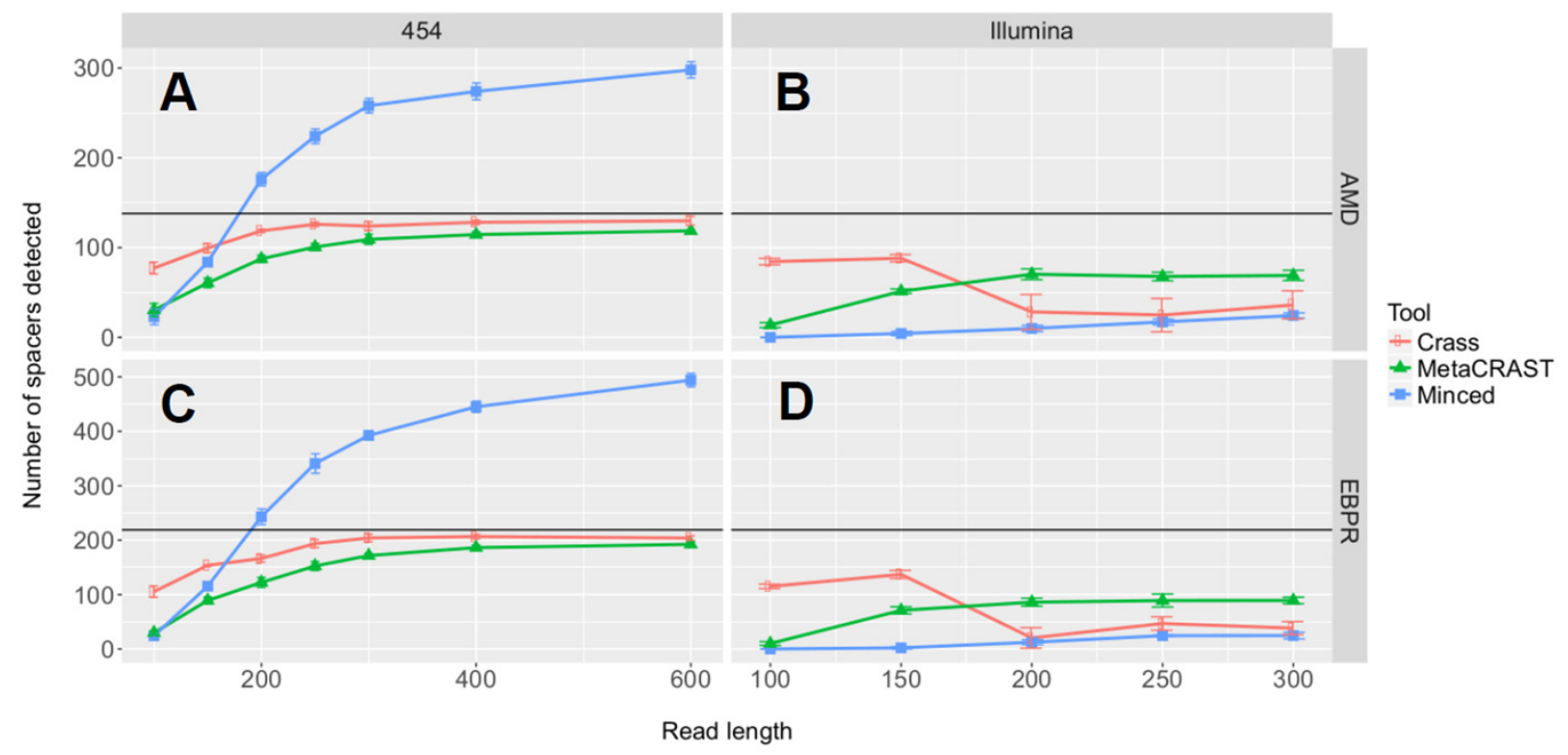

551

552

553

554

555

556 
557 Figure 4: Evaluation of MetaCRAST, Crass, and MinCED performance on simulated 558 metagenomes with varying proportions of Ferroplasma acidarmanus fer1 and 559 Leptospirillum sp. Group II 'CF-1' genome sequences. Simulated metagenomes were 560 generated with Grinder. The data points shown represent the average number of "true 561 positive" spacers detected that matched spacers in corresponding Ferroplasma or 562 Leptospirillum CRISPR arrays (A and B, respectively). All data points represent the 563 averages of six individual simulations and are presented with error bars representing two 564 times the standard error above and two below the average. The true number of spacers 565 expected for each genome is marked with a black line (20 expected in the Ferroplasma 566 genome; 118 in the Leptospirillum genome).

567

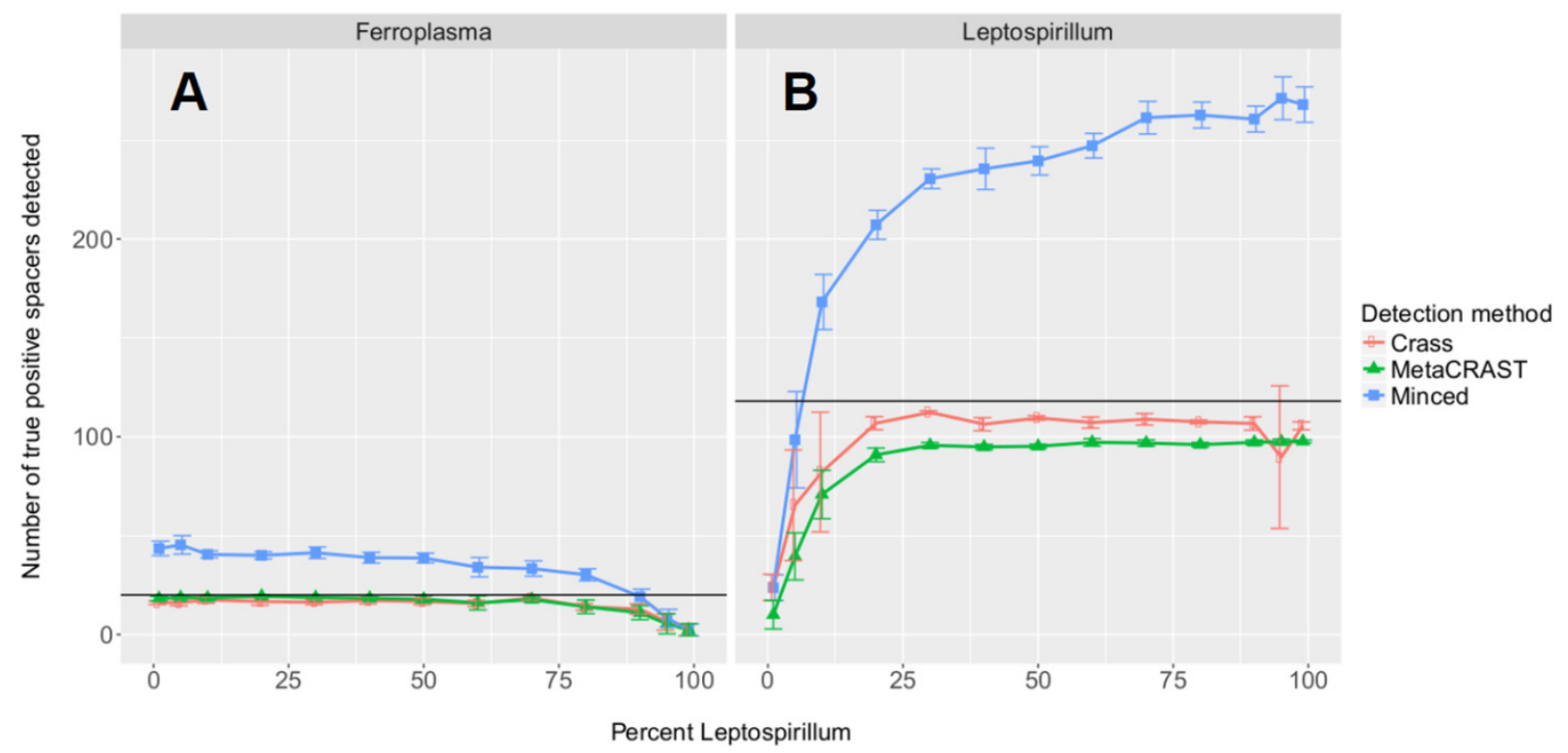

569

570

571

572 
573 Figure 5: Evaluation of MetaCRAST, Crass, and MinCED on real AMD and EBPR 574 metagenomes. A) Total number of CRISPR spacers detected in real AMD and EBPR 575 metagenomes using four different detection methods - Crass (de novo), MetaCRAST 576 (using assembly-guided queries), MetaCRAST (using taxonomy-guided queries), and 577 MinCED (de novo). Taxonomy-guided and assembly-guided queries are provided as 578 Tables S3-S4 and S6-S7. B) Comparison of spacers detected in the real AMD 579 metagenome using Crass (de novo), MetaCRAST (using taxonomy-guided queries), 580 MetaCRAST (using assembly-guided queries), and MinCED (de novo). Comparison was 581 performed using Venny 2.1 (http://bioinfogp.cnb.csic.es/tools/venny/). C) Comparison of 582 spacers detected in the real EBPR metagenome using the same methods as in B. 583 Comparison was performed using Venny 2.1.

A

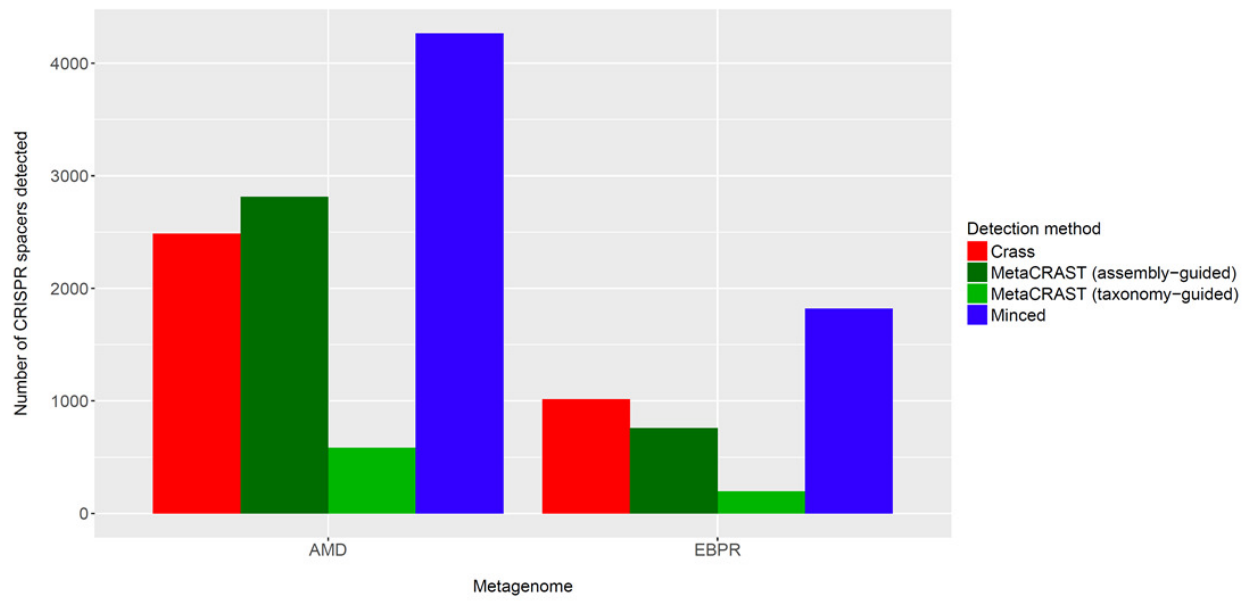

B

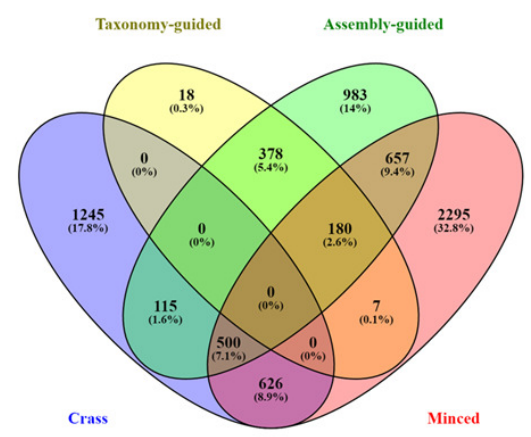

C

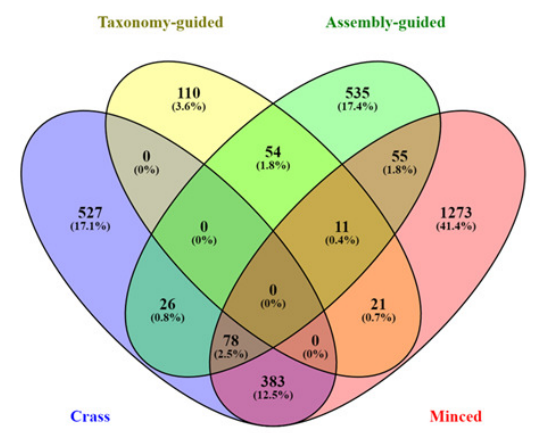


586 Figure 6: Evaluation of MetaCRAST, Crass, and MinCED false positive detection on

587 simulated AMD ( $A$ and $B$ ) and EBPR ( $C$ and $D)$ metagenomes. The procedure for 588 generating the simulated metagenomes is described in Materials and Methods. The 589 number of detected spacers matching expected ones was subtracted from the total 590 number of spacers detected to determine the number of false positive spacers for a 591 particular method and condition. All data points represent the averages of three 592 individual simulations and are presented with error bars representing two times the 593 standard error above and two below the average.

594

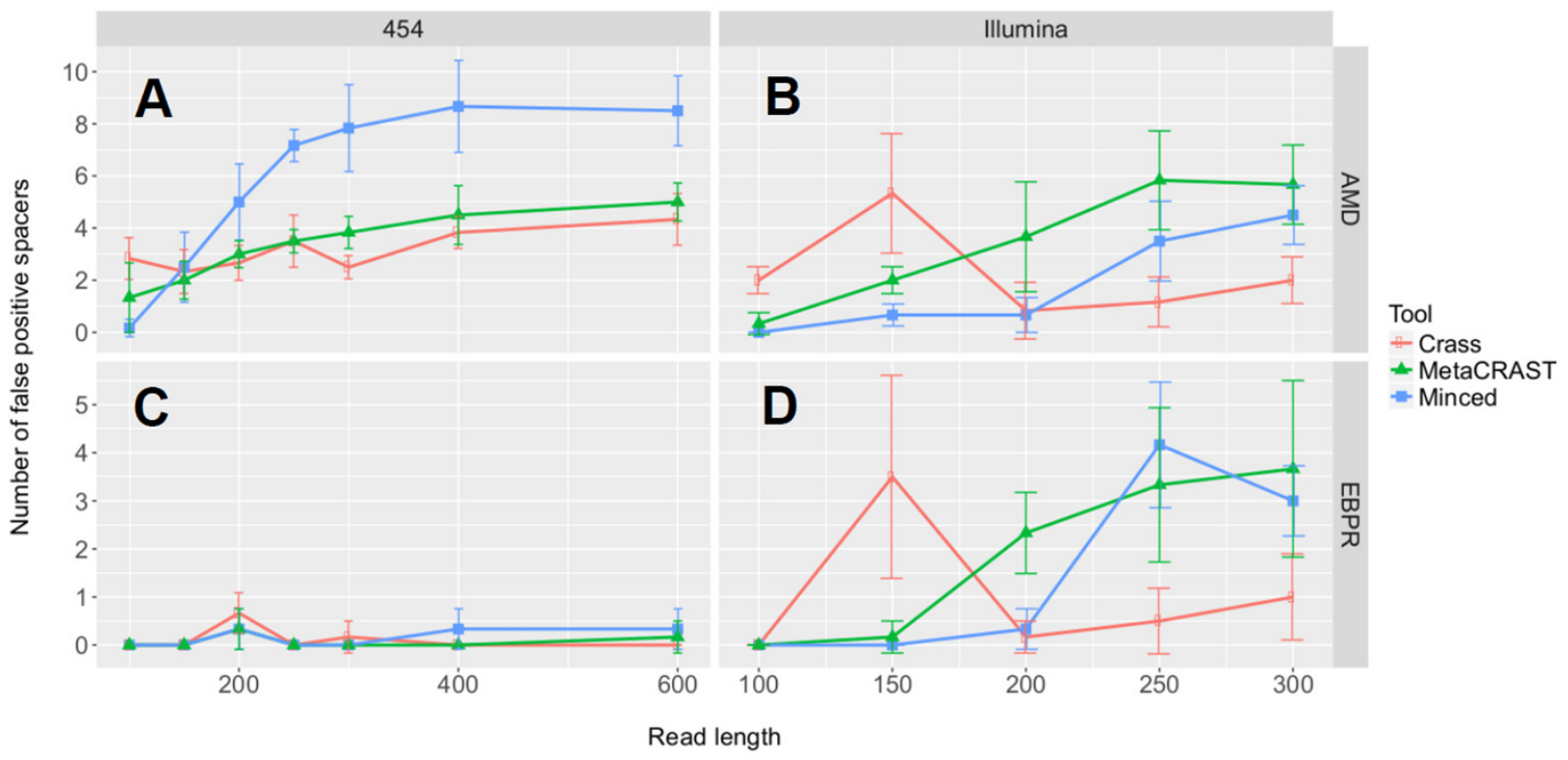

595

596

597

598

599

600 
602 Figure 7: Evaluation of MetaCRAST, Crass, and MinCED run times on simulated AMD (A 603 and $B$ ) and EBPR ( $C$ and $D$ ) metagenomes. We evaluated seven different combinations 604 of algorithms, implementations, and parameters. We evaluated both Crass and MinCED 605 with default parameters. For MetaCRAST, we evaluated five different conditions differing 606 in parallelization and metagenome loading method - BioPerl loading and 16 threads, 607 BioPerl and a single thread, readfq with mce_open for loading and 16 threads, readfq 608 with mce_open and a single thread, and readfq with the standard open routine and a 609 single thread. The procedure for generating the simulated metagenomes is described in 610 Materials and Methods. Run time was calculated as the sum of the user and system time 611 (together the total CPU time). All data points represent the averages of three individual 612 simulations and are presented with error bars representing two times the standard error 613 above and two below the average.

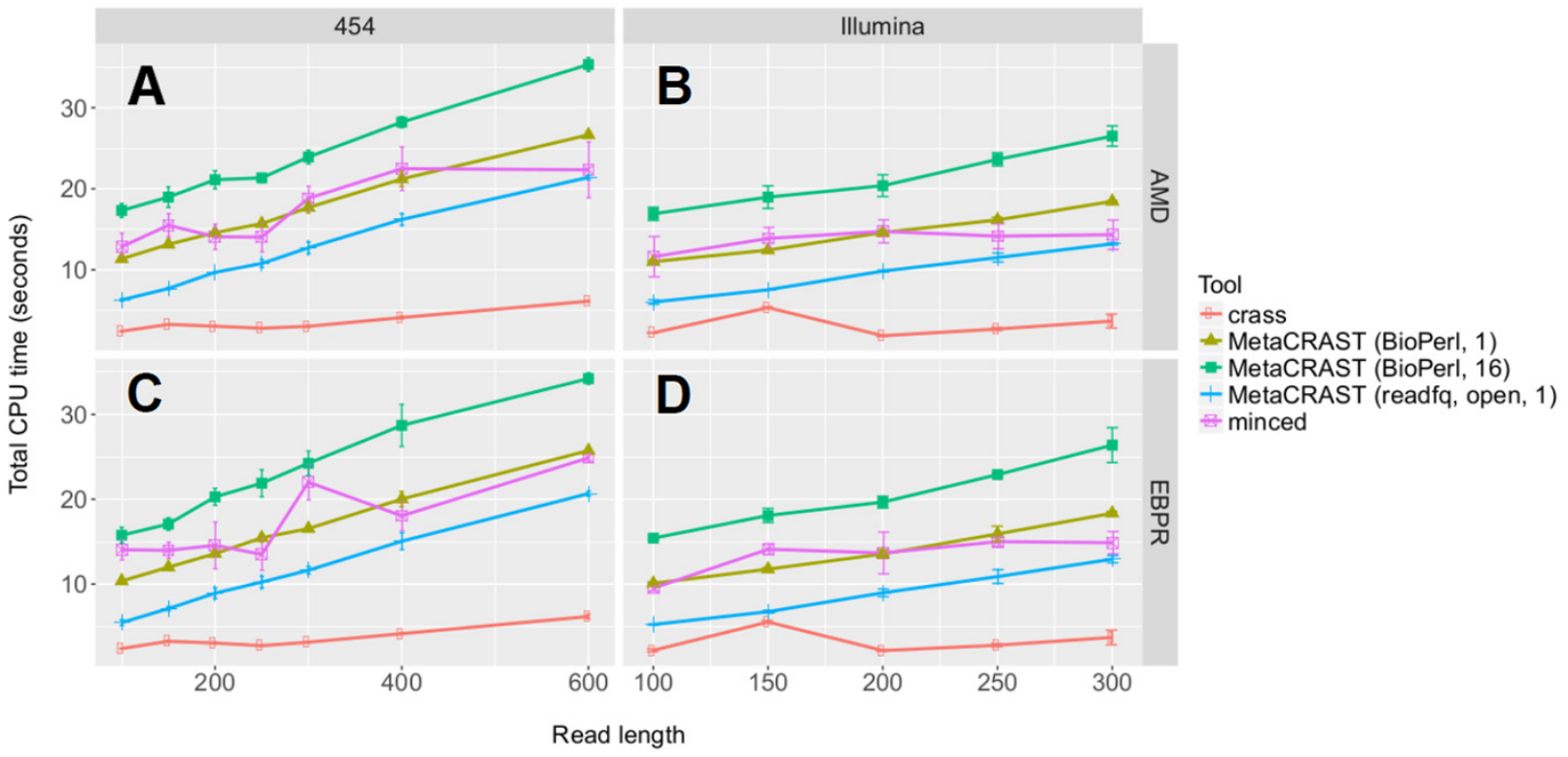

614

615

616

617

618 
620 Table 1: Command line arguments for MetaCRAST. Required arguments are in bold.

\begin{tabular}{|c|c|}
\hline Argument & Description \\
\hline$-p$ & Pattern file containing query DR sequences in FASTA or FASTQ format \\
\hline$-\mathbf{i}$ & Input metagenome in FASTA or FASTQ format \\
\hline-0 & Output directory for detected reads and spacers \\
\hline$-d$ & $\begin{array}{l}\text { Allowed edit distance (insertions, deletions, or substitutions) for initial read } \\
\text { detection with the Wu-Manber algorithm and subsequent DR detection steps }\end{array}$ \\
\hline$-\mathrm{t}$ & $\begin{array}{l}\text { Temporary directory to put metagenome parts (use this if }-n \text { option also } \\
\text { selected) }\end{array}$ \\
\hline$-q$ & $\begin{array}{l}\text { Input metagenome is a FASTQ file (directs use of fastq-splitter.pl instead } \\
\text { of fasta-splitter.pl) }\end{array}$ \\
\hline$-\mathrm{h}$ & $\begin{array}{l}\text { Use Hamming distance metric (substitutions only - no insertions or } \\
\text { deletions) to find direct repeat locations in reads (default: use Levenshtein } \\
\text { distance metric - look for sequences matching DR within insertion, deletion, } \\
\text { and/or substitution edit distance) }\end{array}$ \\
\hline$-\mathrm{r}$ & Search for reverse complement of CRISPR direct repeat sequences \\
\hline-1 & Maximum spacer length in bp \\
\hline$-\mathrm{C}$ & $\begin{array}{l}\text { CD-HIT similarity threshold for clustering spacers detected for each query } \\
\text { direct repeat (value from } 0 \text { to } 1 \text { ) }\end{array}$ \\
\hline$-a$ & $\begin{array}{l}\text { CD-HIT similarity threshold for clustering all detected spacers (value from } 0 \\
\text { to 1) }\end{array}$ \\
\hline$-n$ & $\begin{array}{l}\text { Number of processors to use for parallel processing (and number of } \\
\text { temporary metagenome parts) }\end{array}$ \\
\hline
\end{tabular}


626 Table 2: Distribution statistics for binomial name and genus-level taxonomic affiliation of 627 CRISPRdb direct repeats. A library of direct repeats labeled with respective GenBank 628 accessions from CRISPRdb was processed to assign taxonomy information based on 629 GenBank accession. Taxonomy information was extracted from GenBank records with 630 the Perl module Bio::DB::GenBank. Statistics describing the distribution of binomial 631 names or genuses to which individual direct repeats affiliated were compiled with 632 Microsoft Excel.

\begin{tabular}{|l|l|l|}
\hline Statistic & Binomial names & Genuses \\
\hline Mean & 1.308 & 1.063 \\
\hline Median & 1 & 1 \\
\hline Mode & 1 & 1 \\
\hline Minimum & 1 & 1 \\
\hline Maximum & 46 & 20 \\
\hline Standard deviation & 1.567 & 0.521 \\
\hline
\end{tabular}

